\title{
Homogeneous Carbon Nanotube/Carbon Composites Prepared by Catalyzed Carbonization Approach at Low Temperature
}

\author{
Hongjiang Li, Changhong Liu, and Shoushan Fan \\ Department of Physics, Tsinghua-Foxconn Nanotechnology Research Center, Tsinghua University, Beijing 100084, China \\ Correspondence should be addressed to Changhong Liu, chliu@tsinghua.edu.cn
}

Received 17 May 2010; Revised 17 August 2010; Accepted 26 August 2010

Academic Editor: Jianyu Huang

Copyright () 2011 Hongjiang Li et al. This is an open access article distributed under the Creative Commons Attribution License, which permits unrestricted use, distribution, and reproduction in any medium, provided the original work is properly cited.

\begin{abstract}
We synthesize carbon nanotube (CNT)/carbon composite using catalyzed carbonization of CNT/Epoxy Resin composite at a fairly low temperature of about $400^{\circ} \mathrm{C}$. The microstructure of the composite is characterized by scanning electron microscope (SEM), transmission electron microscope (TEM), and X-ray diffraction (XRD). The results indicate that CNTs and pyrolytic carbon blend well with each other. Pyrolytic carbon mainly stays in an amorphous state, with some of it forming crystalline structures. The catalyst has the effect of eliminating the interstices in the composites. Remarkable increases in thermal and electrical conductivity are also reported.
\end{abstract}

\section{Introduction}

Owing to the superior thermal, electrical, and mechanical properties, carbon nanotube (CNT) has been widely used in composite materials, especially in polymer based composites [1-9]. Carbon nanotube/carbon (CNT/C) is a promising new composite to make a better contact between CNT and the matrix material. For one thing, CNT and carbon have the same constituent atoms, which will tend to make a better connection between them. For another, carbon itself is a kind of material with better conduct properties than most of polymers, so the CNT/C composite has the potential to gain higher conducting performance than $\mathrm{CNT} /$ polymer composites.

Some attempts have already been made on the synthesis of CNT/C composites; many of which are inspired by the processing methods of carbon/carbon $(\mathrm{C} / \mathrm{C})$ composites, using either chemical vapor infiltration (CVI) method [10, 11] or high pressure impregnation carbonization method [12-15]. High temperature or high pressure is needed in such processes. In the field of application, CNT/C composites can be utilized in many aspects, as structural or functional materials. Researchers have added CNTs into carbon to promote the tribological behavior $[10,16]$, to improve the absorption property $[17,18]$, to make electrodes $[19,20]$ for electrochemistry, and so on.
In our previous work [21], we proposed a facile postprocessing way to fabricate aligned CNT array/carbon composites at relatively low temperature and pressure. In this paper, we follow the in-situ method, using catalyzed carbonization of organic material to make homogenous CNT/C composite, making it a promising method for largescale production. Instead of aligned CNT array and glucose, we utilize dispersed multiwalled CNTs and epoxy resin in the process, making it a more general situation. We believe that by this method the material structures can be tuned a lot. We did contrast experiment to analyze the role of the catalyst in the carbonization process. In addition, we measured the thermal properties of the composite and found a remarkable increase in the thermal conductivity and decrease in the contact thermal resistance. Electrical conducting property is also tested.

\section{Experimental}

The raw multi-walled CNTs were grown by chemical vapor deposition (CVD). The purity of the CNTs is $95 \%$, with diameter about $20 \mathrm{~nm}$ and length around $10 \mu \mathrm{m}$. Epoxy resin is chosen as carbon resource to produce pyrolytic carbon. The catalyst of carbonization is ferrocene. We first synthesize CNT/Epoxy resin composite, with around $2 \mathrm{wt} \%$ of 


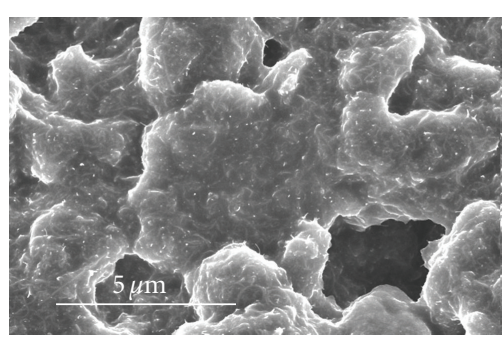

(a)

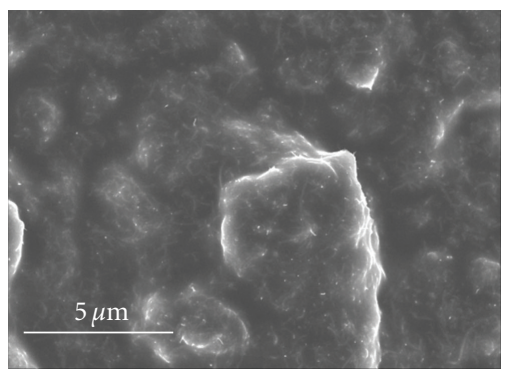

(b)

Figure 1: SEM top view of as-prepared CNT/C composites (a) without and (b) with ferrocene catalyst.

multi-walled CNTs and $0.1 \mathrm{wt} \%$ of ferrocene. The process is as follows: we put CNTs into epoxy monomer and add some ethanol to enhance fluidity. After that the mixture is stirred for 1 hour before another hour of ultrasonic treatment. Then it is cooled down and added into the catalyst and curing agent, which in our case is polyamide resin. Another hour of stirring will be needed before we put the mixture into the mold and wait about 12 hours for complete curing. Then the sample was heated in a vacuum tube furnace, which was gradually elevated to $400^{\circ} \mathrm{C}$ from room temperature and maintained this temperature for 1 hour before cooling. Nitrogen atmosphere was used in this treatment to protect the sample from oxidation. We had tried this temperature in the range $200-600^{\circ} \mathrm{C}$. We found that $400^{\circ} \mathrm{C}$ was more favorable.

Scanning electron microscopy (SEM, Sirion 200, resolution $1.0 \mathrm{~nm}$ ) and high resolution transmission electron microscopy (HRTEM, Tecnai G2 F20 S-Twin, resolution $2.4 \mathrm{~nm}$ ) were used for characterization of microstructures. Thermal conductivity and contact resistance were measured by the static method, using an apparatus designed according to ASTM D5470, which was described elsewhere [8]. The samples have two kinds of shapes, plates with a diameter of $11.5 \mathrm{~mm}$ and $25 \mathrm{~mm}$. They have different thickness, ranging from $0.5 \mathrm{~mm}$ to $2 \mathrm{~mm}$. Electrical conductivity was measured by a four-point method, using Keithley's Model 2410 HighVoltage SourceMeter.

\section{Results and Discussion}

Since the temperature of carbonization was fairly low, the weight loss of the material was as low as about $20 \%$. The density of the obtained CNT/C sample was $0.98 \mathrm{~g} / \mathrm{cm}^{3}$, which was remarkably higher than the typical pristine CNTs tablet $\left(0.65 \mathrm{~g} / \mathrm{cm}^{3}\right)$. The enhancement in density shows that pyrolytic carbon fills the interstices between the CNTs, and instead of increasing the volume, the pyrolytic carbon holds the CNTs closer, forming a much denser material.

The microstructure of as-prepared CNT/C composites is shown in Figure 1. The sample in Figure 1(a) is synthesized by directly carbonizing CNT/Epoxy resin while the other sample made by catalyzed carbonization of CNT/Epoxy resin is shown in Figure 1(b), with ferrocene as a catalyst. We can see from the two pictures that CNTs blend well with the pyrolytic carbon, forming block structures. The dispersed CNTs are buried under the surface of pyrolytic carbon, establishing the framework of the blocks while the pyrolytic carbon closely surrounds the CNTs, making a compact compound structure. Such blocks stack with one another, building up the composite. The catalyst, ferrocene, plays an important role in the formation of the composite. In comparison Figures 1(a) and 1(b), we can see that there are many gaps between the blocks in Figure 1(a); however, with catalyst in Figure 1(b), such gaps disappear. So the ferrocene can improve the connections between the blocks, which will be beneficial to the conduct properties of the material. All the CNT/C composites talked about afterwards are made by catalyzed carbonization.

Figure 2 is the TEM image of the CNT/C composite. The close contact between the CNT and pyrolytic carbon is shown in Figure 2(a). We can see that the CNT is surrounded by the pyrolytic carbon; most of which is in an amorphous state. Their connection is so strong that we can hardly find the interface between them. In order to prepare the TEM sample uniformly, we treated the composite flake with ultrasonic for more than 2 hours; the power of which is $500 \mathrm{~W}$. However, it is still hard to separate the CNT and pyrolytic carbon apart. Most of them intertwine together, forming big blocks in the pictures. It shows that the two components have strong interaction between them. Figure 2(b) gives us an enlarged view of the pyrolytic carbon. Most of the pyrolytic carbon is in an amorphous state, but on the edge of the sample, we can see some crystal texture (shown in the inset picture), where the interplaner spacing is about $0.34 \mathrm{~nm}$, the same as graphite. Unfortunately, such crystalline structures do not extend over a long distance. Because of the large amount of amorphous carbon, and the strong interaction between the two components, it is hard for us to find more CNT feature under the cover of amorphous carbon.

X-ray diffraction (XRD) pattern of MWNT and MWNT/ $\mathrm{C}$ are presented in Figure 3. The characteristic peak of MWNT is around 25.9 degree. For the latter, the peak is widened a lot, which indicates the existence of a large amount of small particles of amorphous carbon. This result is consistent with the microstructure characterization. Besides, although the MWNT is only a little proportion in the composite, the characteristic peak is still seen, showing that the CNTs maintain their structure well after carbonization process.

We measured the thermal properties of three different samples: MWNT/Epoxy Resin composite, MWNT tablet and 


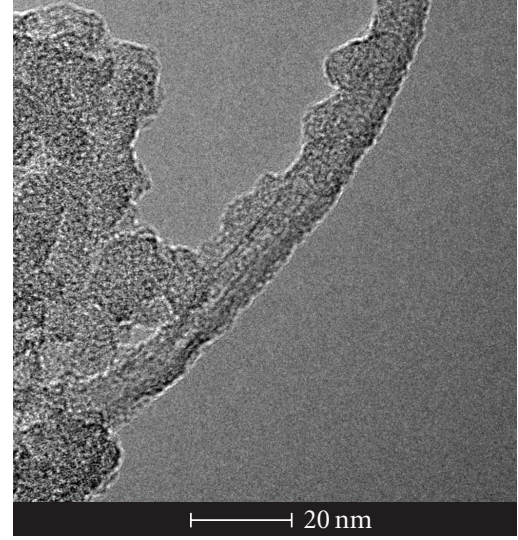

(a)

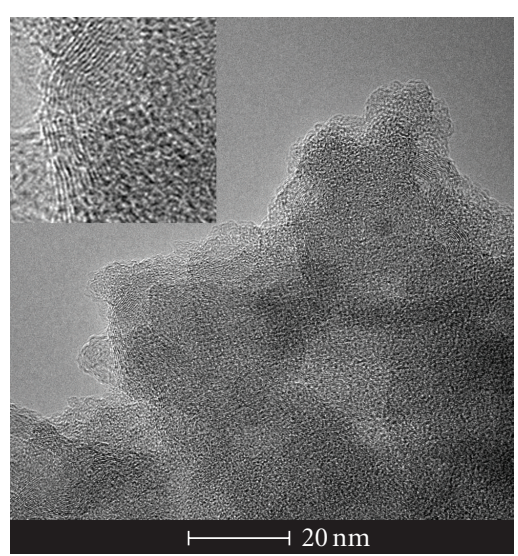

(b)

FIGURE 2: TEM image of CNT/C composite: (a) the image showing the close contact of CNT and pyrolytic carbon. (b) the microstructure of the pyrolytic carbon. The inset shows an enlarged feature of the image.

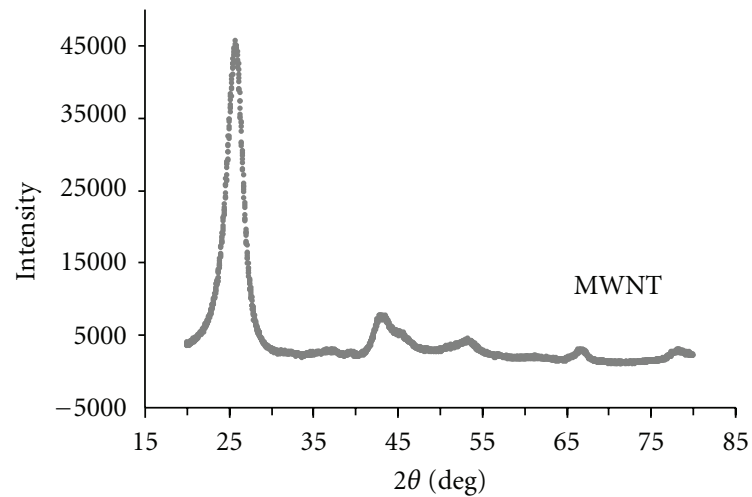

(a)

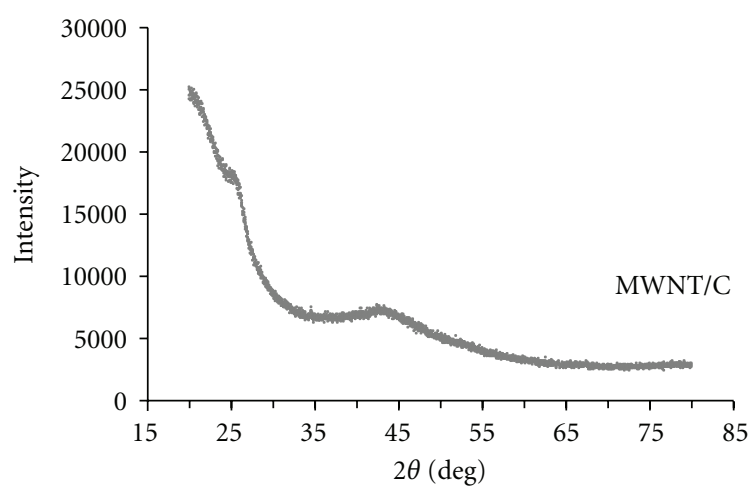

(b)

FIGURE 3: The XRD patterns of (a) MWNT tablet and (b) the MWNT/C composite. The characteristic peak of the MWNTs was around 25.9 degree.

MWNT/C composite. The thermal conductivity and thermal contact resistance are shown in Figure 4. The shown data was the average of multiple tests (three times) for each sample, and the standard deviation for the results was below $10 \%$. The white bars represent the thermal conductivity while the black ones represent the thermal contact resistance.

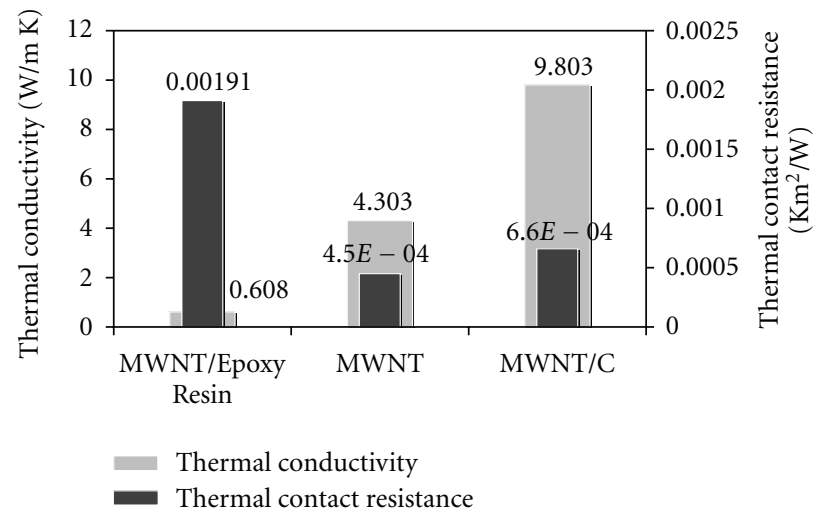

FIGURE 4: The thermal conductivity and thermal contact resistance of MWNT/Epoxy Resin composite, MWNT tablet, and MWNT/C composite. The white bars represent thermal conductivity, while the black ones are the thermal contact resistance.

From the white bars, we see that the thermal conductivity of MWNT/C composites, which is more than twice the value of pure MWNT tablet, can increase remarkably by one magnitude than MWNT/Epoxy Resin composite. This increase is attributed to the good connections between the CNTs and pyrolytic carbon as well as the relatively high thermal conductivity of pyrolytic carbon, which, instead of the air, fills in the interstices among the CNTs. The black bars give us evident information that the thermal contact resistance of $\mathrm{MWNT} / \mathrm{C}$ composites remains a small value, a little higher than pure MWNT but only about $1 / 3$ of the value of MWNT/Epoxy Resin composite. We believe that the amorphous carbon in the pyrolytic carbon plays an important role in decreasing of the thermal contact resistance, for the small blocks can easily get into the gaps between the two testing probes, squeeze the air in them, and get a better thermal connection.

The MWNT/C composite has much higher thermal conductivity and fairly low thermal contact resistance, which 
is appropriate for thermal interface materials (TIMs). TIMs are important materials for thermal conduction between interfaces and are being wildly used in electronic packing. As the electronics and information industry is progressing, higher standard of thermal conduction will be needed for a higher integrated density. MWNT/C composites are one kind of potential material for future thermal conduction. Besides, although the controlled growth of CNTs is still being studied, MWNTs are easily fabricated by CVD method, which will help to reduce the cost of this industry.

In addition, we measured the electrical resistivity of the MWNT/Epoxy Resin and MWNT/C composites. The value decreased a little from $3.1 \times 10^{7} \Omega \cdot \mathrm{m}$ to $2.5 \times 10^{6} \Omega \cdot \mathrm{m}$ after the carbonization. It shows that pyrolytic carbon has a better electrical conducting property than Epoxy Resin. However, because of the low carbonization temperature, the increase in conductivity is not so remarkable. Other characterizations are still on the way.

\section{Conclusions}

In this paper, we fabricate MWNT/C composites by the method of catalyzed carbonization of MWNT/Epoxy Resin composite. The microstructure shows that the CNTs and the pyrolytic carbon make a compact compound material, with CNTs and carbon evenly distributed. The catalyst, ferrocene, plays an important role in making a denser composite structure. From the high-resolution TEM images, we see that pyrolytic carbon wraps around the MWNTs. Pyrolytic carbon is made up of amorphous carbon and crystal carbon, with amorphous carbon as the majority. The crystalline structure can be found on the edge of the amorphous carbon. The measurements of thermal properties of this composite indicate that the MWNT/C composite has a much higher thermal conductivity and a fairly low thermal contact resistance, which make it a good choice for the TIMs. The electrical conducting properties of the composite are also improved, increasing by one order of magnitude as to CNT/Epoxy Resin composite.

\section{Acknowledgments}

This paper was supported by the National Basic Research Program of China (2005CB623606) and the National Natural Science Foundation of China (50673049).

\section{References}

[1] B.-K. Zhu, S.-H. Xie, Z.-K. Xu, and Y.-Y. Xu, "Preparation and properties of the polyimide/multi-walled carbon nanotubes (MWNTs) nanocomposites," Composites Science and Technology, vol. 66, no. 3-4, pp. 548-554, 2006.

[2] Q. Zhang, S. Rastogi, D. Chen, D. Lippits, and P. J. Lemstra, "Low percolation threshold in single-walled carbon nanotube/high density polyethylene composites prepared by melt processing technique," Carbon, vol. 44, no. 4, pp. 778-785, 2006.
[3] E. T. Thostenson and T.-W. Chou, "Processing-structuremulti-functional property relationship in carbon nanotube/ epoxy composites," Carbon, vol. 44, no. 14, pp. 3022-3029, 2006.

[4] X. J. He, J. H. Du, Z. Ying, and H. M. Cheng, "Positive temperature coefficient effect in multiwalled carbon nanotube/highdensity polyethylene composites," Applied Physics Letters, vol. 86, no. 6, Article ID 062112, 3 pages, 2005.

[5] A. R. Bhattacharyya, T. V. Sreekumar, T. Liu et al., "Crystallization and orientation studies in polypropylene/single wall carbon nanotube composite," Polymer, vol. 44, no. 8, pp. 2373-2377, 2003.

[6] R. Haggenmueller, H. H. Gommans, A. G. Rinzler, J. E. Fischer, and K. I. Winey, "Aligned single-wall carbon nanotubes in composites by melt processing methods," Chemical Physics Letters, vol. 330, no. 3-4, pp. 219-225, 2000.

[7] R. Andrews, D. Jacques, A. M. Rao et al., "Nanotube composite carbon fibers," Applied Physics Letters, vol. 75, no. 9, pp. 13291331, 1999.

[8] H. Huang, C. Liu, Y. Wu, and S. Fan, "Aligned carbon nanotube composite films for thermal management," Advanced Materials, vol. 17, no. 13, pp. 1652-1656, 2005.

[9] C. H. Liu, H. Huang, Y. Wu, and S. S. Fan, "Thermal conductivity improvement of silicone elastomer with carbon nanotube loading," Applied Physics Letters, vol. 84, no. 21, pp. 4248-4250, 2004.

[10] Q.-M. Gong, Z. Li, Z. Zhang et al., "Tribological properties of carbon nanotube-doped carbon/carbon composites," Tribology International, vol. 39, no. 9, pp. 937-944, 2006.

[11] Q.-M. Gong, Z. Li, D. Li, X.-D. Bai, and J. Liang, "Fabrication and structure: a study of aligned carbon nanotube/carbon nanocomposites," Solid State Communications, vol. 131, no. 6, pp. 399-404, 2004.

[12] B. Wu, Z. Wang, Q. M. Gong, H. H. Song, and J. Liang, "Fabrication and mechanical properties of in situ prepared mesocarbon microbead/carbon nanotube composites," Materials Science and Engineering A, vol. 487, no. 1-2, pp. 271-277, 2008.

[13] Z. Wang, B. Wu, Q. Gong, H. Song, and J. Liang, "In situ fabrication of carbon nanotube/mesocarbon microbead composites from coal tar pitch," Materials Letters, vol. 62, no. 20, pp. 3585-3587, 2008.

[14] Y. Song, G. Zhai, J. Shi, Q. Guo, and L. Liu, "Carbon nanotube: carbon composites with matrix derived from oxidized mesophase pitch," Journal of Materials Science, vol. 42, no. 22, pp. 9498-9500, 2007.

[15] Z. Li, Q.-M. Gong, Z.-Y. Zhang, J. Liang, Q.-Z. Huang, and B.-Y. Huang, "Fabrication and characterization of a new pitchbased ACNT/C nanocomposites," Chinese Journal of Inorganic Chemistry, vol. 22, no. 10, pp. 1755-1760, 2006.

[16] Q.-M. Gong, Z. Li, X.-D. Bai, D. Li, Y. Zhao, and J. Liang, "Thermal properties of aligned carbon nanotube/carbon nanocomposites," Materials Science and Engineering A, vol. 384, no. 1-2, pp. 209-214, 2004.

[17] K. Dai, L. Shi, D. Zhang, and J. Fang, "NaCl adsorption in multi-walled carbon nanotube/active carbon combination electrode," Chemical Engineering Science, vol. 61, no. 2, pp. 428-433, 2006.

[18] C. Ye, Q.-M. Gong, F.-P. Lu, and J. Liang, "Preparation of carbon nanotubes/phenolic-resin-derived activated carbon spheres for the removal of middle molecular weight toxins," Separation and Purification Technology, vol. 61, no. 1, pp. 914, 2008. 
[19] J. Luo, J. Zhu, Z. Huang, and L. Zhang, "Arrays of $\mathrm{Ni}$ nanowire/multiwalled carbon nanotube/amorphous carbon nanotube heterojunctions containing Schottky contacts," Applied Physics Letters, vol. 90, no. 3, Article ID 033114, 2007.

[20] P. Deng, J. Fei, J. Zhang, and J. Li, "Determination of trace copper by adsorptive voltammetry using a multiwalled carbon nanotube modified carbon paste electrode," Electroanalysis, vol. 20, no. 11, pp. 1215-1219, 2008.

[21] H. Li, C. Liu, and S. Fan, "Catalyzed filling of carbon nanotube array with graphite and the thermal properties of the composites," Journal of Physical Chemistry C, vol. 112, no. 15, pp. 5840-5842, 2008. 

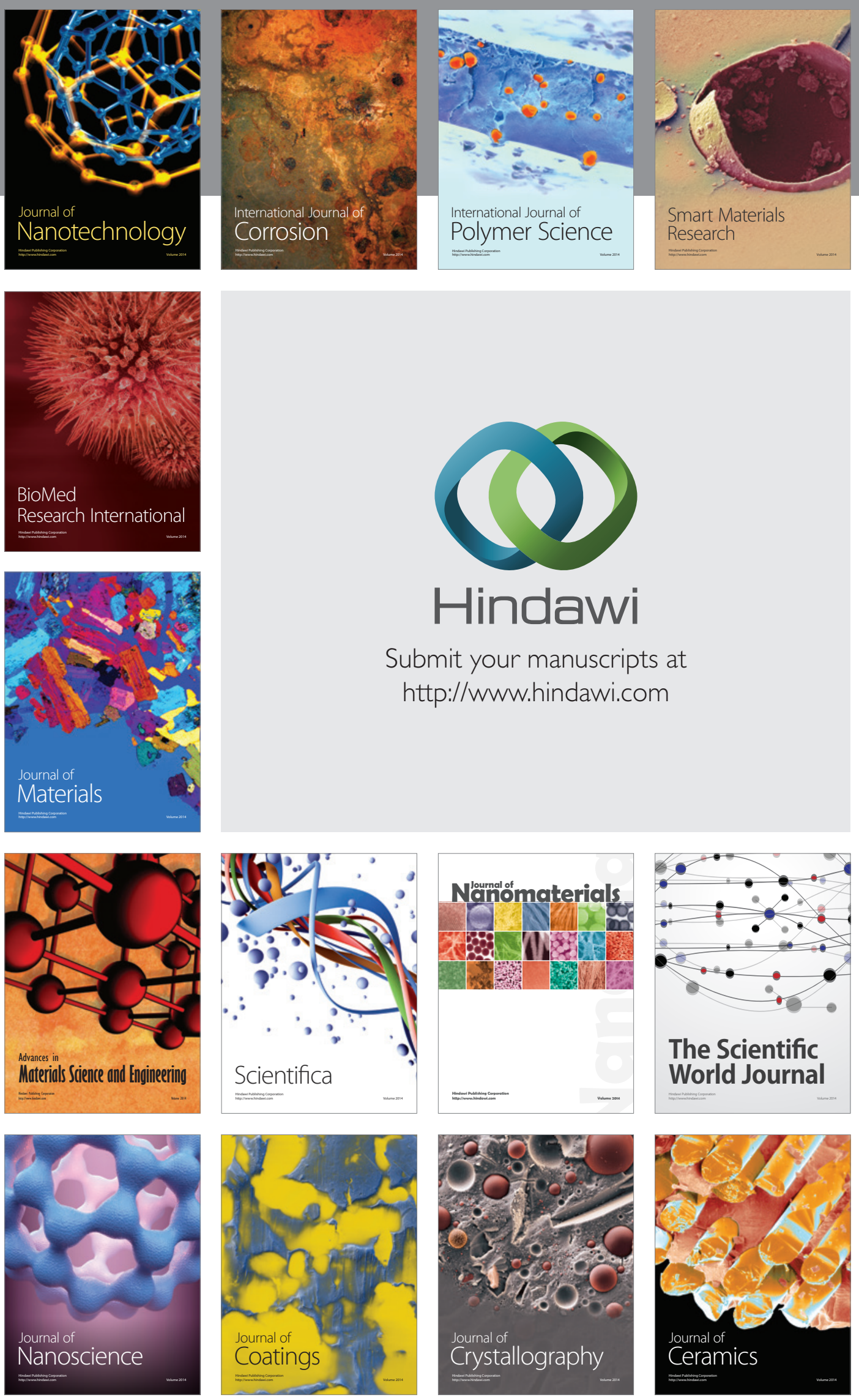

The Scientific World Journal

Submit your manuscripts at

http://www.hindawi.com

\section{World Journal}

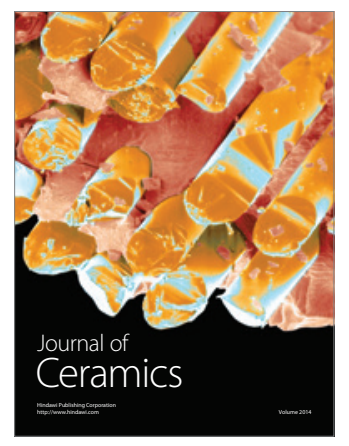

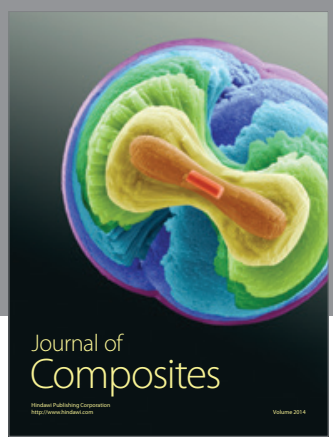
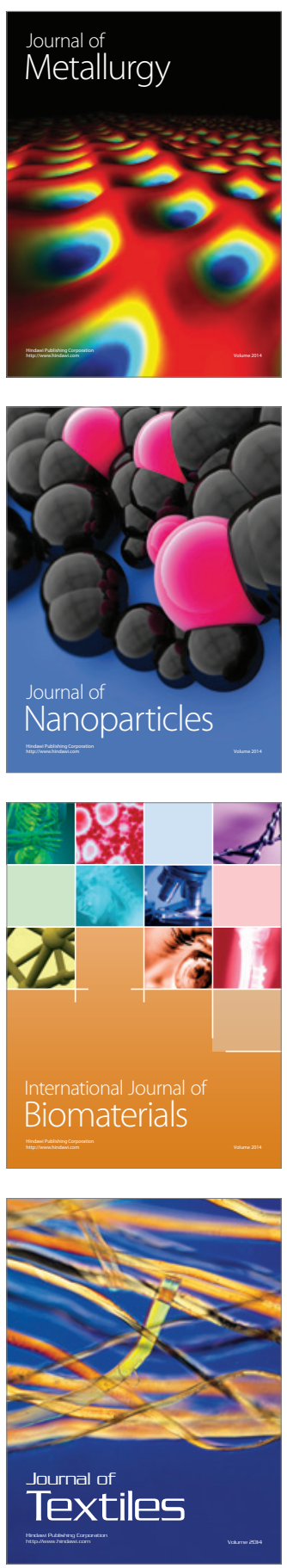